\title{
Sur la distribution géographique de la faune interstitielle du Danube et de certains de ses affluents en Basse-Autriche
}

\author{
par
}

DAN L. DANIELOPOL

On the geographical distribution of the interstitial fauna of the Danube and some of its tributaries in Lower-Austria.

\section{SUMMARY}

The wide distribution of the interstitial isopod Proasellus slarus as well as new records of Troglochaetus (Archiannelida) and Bogidiella (Amphipoda) in Lower-Austria are presented. Species of the genera Microcharon (Isopoda Microparasellidae), Koralevskiella (Ostracoda Metacyprinae) and of the family Parabathynellidae (Bathynellacea) are recorded for the first time in Austria.

Les investigations entreprises entre 1973 et 1976 sur les habitats interstitiels, de la vallée du Danube, des plages à graviers et sable longeant le fleuve, entre Ybbs-Persenbeug et Hainburg (voir Danielopol, 1976) ainsi que sur le Piesting en amont de Moosbrunn, le Schwechat en amont de Baden et le Seebach-Ybbs, près de la Station Biologique de Lunz-am-See, m'ont permis de mettre en évidence une riche faune stygobie représentée par des Isopodes, des Amphipodes, des Ostracodes, des Harpacticoïdes, des Limnohalacariens. Ces investigations ont permis, d'une part d'élargir nos connaissances sur les aires de répartition de certaines espèces déjà signalées dans une ou deux stations en Basse-Autriche, d'autre part de trouver des représentants nouveaux pour la faune d'Autriche.

L'Isopode Proasellus slav'us était connu dans le Basse-Autriche seulement d'une seule station (puits à Vienne). Dans la région une deuxième espèce d'Isopode interstitiel, Proasellus strouhali, a été citée comme très fréquente. Vornatscher (1974) écrit à ce sujet: "Das vor dreissig Jahren so seltene Tier ist

^ Limnologisches Institut der Österreichischen Akademie der Wissenschaften. Berggasse 18 , A-I090 WIEN. 
zu unseren häufigsten und verbreitetsten Grundwasserbewohnern geworden." (p. 668). La figure I montre les stations citées par Strouhal (1958) et Vornatscher (1974). Il est à remarquer que Strouhal cite la présence de Pr. strouhali comme "très probable" dans les stations Anninger im Büchenbrünnl, Bisamberg, Mausrodhöhle à Lunz-am-See. Les observations que jai pu faire montrent que $\mathrm{Pr}$. slavus est une espèce largement répandue en BasseAutriche (l'identification en a été faite par Dr. B. Sket, de l'Université de Ljubliana, à qui j'adresse mes vifs remerciements), tandis que Pr. strouhali a, probablement, une distribution discontinue et restreinte dans cette région. Il

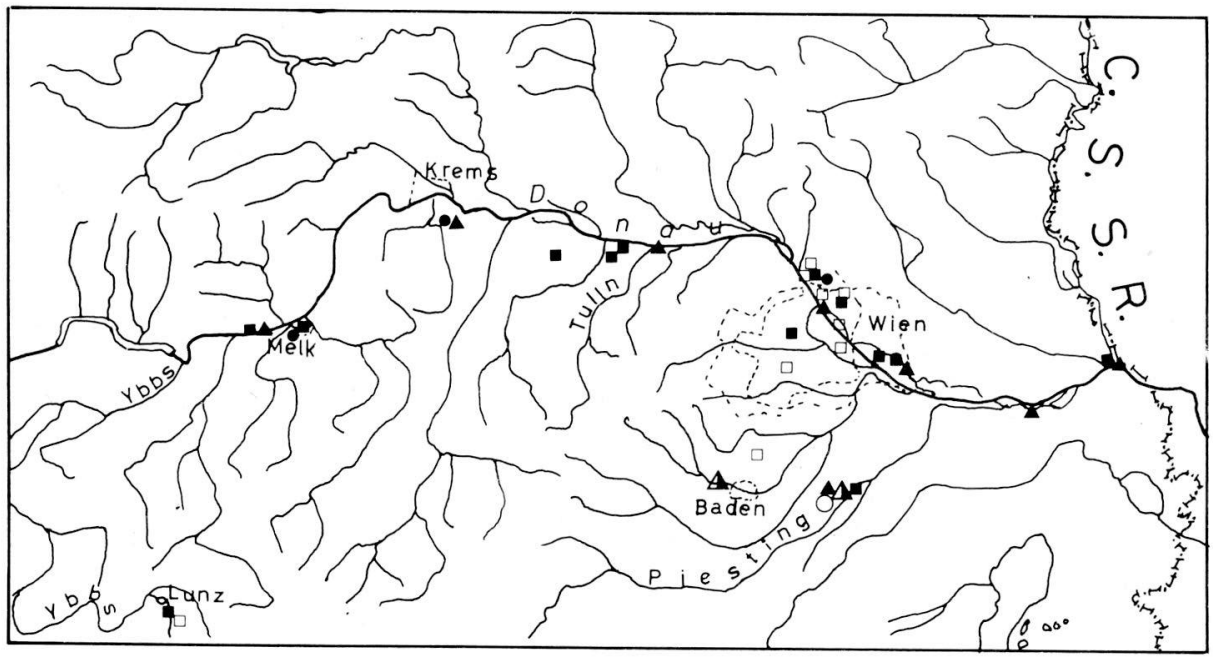

Fig. 1 - Répartition en Basse-Autriche de: Proasellus slavus ( $)$, Pr. strouhali ( $\square$ ) d'après Strouhal (1958) et Vornatscher (1974). Troglochaetus sp. ( - ), Bogidiella sp. (D), Kovalevskiella sp. ( $\Delta$ ) et Parabathynellidae ( $\Delta)$, Microcharon sp ( $O$ ).

est à remarquer qu' aucun exemplaire de cette dernière espèce n'a été trouvé pendant mes trois années d'investigations. La figure 1 montre que Pr. slavus habite les alluvions du lit des rivières ainsi que ceux des vallées. Il est largement répandu le long du Danube et il apparaît aussi sur le Piesting et Seebach-Ybbs. Dans un travail antérieur (1976) j'ai donné une description des stations où cette espèce a été rencontrée. Les stations suivantes sont mentionnées ici pour la première fois: $\left[1^{\circ}\right.$ ) Eberschüttwasser (bras mort) du Danube, dans le Lobau, près de Vienne), dans la proximité de la Kreuzgrundtraverse, à 0,50-0,70 m profondeur sous le fond du "lac". L'échantillon a été prélevé avec une pompe Bou-Rouch en mai 1975 après une période de hautes eaux; les sédiments prélevés en même temps que les animaux sont constitués surtout par du sable fin. Des visites ultérieures de cette station n'ont plus 
révélé d'Isopodes, par contre on les a trouvés dans une sonde piézométrique (T3) située à $50 \mathrm{~m}$ de la station précédente et à $20 \mathrm{~m}$ du rivage du lac. L'eau de cette sonde provient en grande partie d'horizons à graviers. $\left[2^{\circ}\right.$ ) pompes Norton à Vienne: a) dans la région du Lobau en amont de l'Ölhafen, vis à vis du port d'hiver, à 20-30 m environ du bord du Danube et b) dans le quartier Kagran à $500 \mathrm{~m}$, à l'Est de l'église St. Wendelin. Le tube de la pompe de cette dernière station est enfoncé à environ $7 \mathrm{~m}$ de profondeur. $\left[3^{\circ}\right.$.) source captée à $14 \mathrm{~m}$ sous terre, qui a été filtrée pendant plusieurs jours, dans une galerie du futur métro (U-Bahn) à Vienne, près de la Cathédrale St-Stéphane. [4.) Pompe

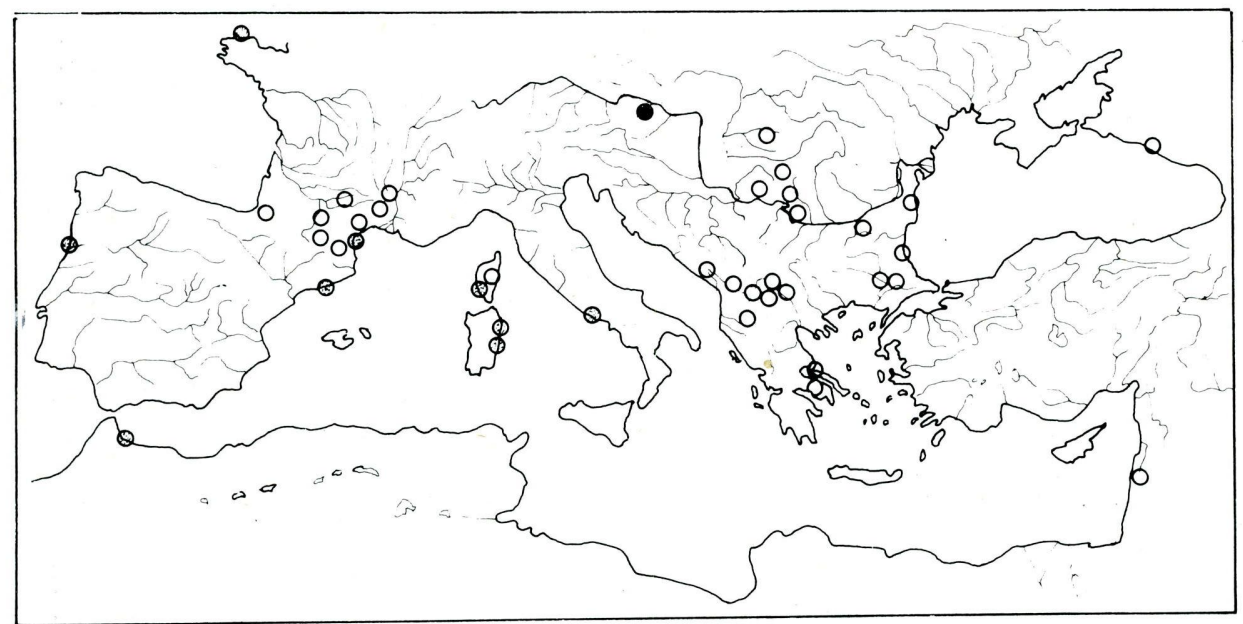

Fig. 2 - Répartition des espèces du genre Microcharon en Europe d’après Coineau (1971) et données inédites. -station litorale marine, -habitats interstitiels des plages litorales marines, $\bigcirc$-habitats hypogés dulçaquicoles, -Microcharon sp. des environs de Vienne.

Norton dans la ville de Melk. [5 $5^{\circ}$.) Sondages faits avec la pompe Bou-Rouch dans les sédiments du Seebach, affluent de l'Ybbs, à Lunz-am-See, en face de la station biologique. Des Isopodes ont été récoltés régulièrement à des profondeurs allant de $0,50 \mathrm{~m}$ à $1,40 \mathrm{~m}$.

De nouvelles stations pour des animaux appartenant aux groupes d'origine marine, Troglochaetus et Bogidiella ont été trouvées ces dernières années. Troglochaetus (Tr. beranecki) était connu en Basse-Autriche d'un puits de Vienne (Vornatscher, 1974) et d'un puits de Melk (Husmann, 1962). Des représentants du genre Bogidiella (B. albertimagni) ont été découverts par Vornatscher (1965) dans un puits à Vienne. Nous avons récolté des Troglochaetus dans un puits à Melk, un puits $(\mathrm{F}-1332)$ à Thallern et un puits à Vienne (Fl). Pour la localisation voir figure l ci-contre et le tableau nr. 2 dans 
Danielopol (1976). Des Bogidiella ont été trouvés dans un sondage fait avec un pompe Bou-Rouch sous le lit du Schwechat en amont de Baden près de la Cholera Kapelle et sur le Piesting dans une sonde piézométrique à $10 \mathrm{~m}$ distance du ruisseau et $6 \mathrm{~m}$ de profondeur, en face de la station de pompage d'eau Moosbrunn II (voir aussi Danielopol, 1976 et figure 1).

Nous avons rencontré pour la première fois en Autriche des Isopodes Microparasellidae (Microcharon sp.) dans des sondes piézométriques qui longent le Piesting dans le périmètre de la sonde à Bogidiella sp. Les représentants du genre Microcharon étaient connus dans le bassin du Danube seulement dans sa partie orientale, i.e. en Roumanie: dans des localités près des rivières Crisu Repede et Strei (en Transylvanie), près de la Nera et Minis (en Banat), sur le Motru (en Olténie), ainsi que le long du Danube à Virciorova et Ostrovu Mare (voir pour plus de détails Serban, 1966; les deux dernières stations sont citées ici pour la première fois d'après le matériel récolté par Serban, Dancau et Danielopol). En Bulgarie Cvetkov (1967) a trouvé des Microcharon près de la ville de Russe et dans la vallée de Jantra près de Velichkovo. Par ailleurs en Europe ce genre d'Isopode interstitiel est largement répandu autour du bassin méditerranéen ainsi que sur les côtes de l'Atlantique. La figure 2 présente la distribution géographique du genre Microcharon en Europe d'après mes propres données et celles de Coineau (1971).

De nouveaux animaux stygobies montrant les affinités de la faune du bassin danubien d'Autriche avec celle de la région orientaie du bassin du Danube et de la péninsule balkanique ont été découverts depuis 1973. Parmi les Ostracodes hypogés ceux du genre Kovalevskiella (=Cordocythere) sont répandus dans l'Est et le Sud-Est de l'Europe (Danielopol, 1970 et données inédites concernant leur présence dans un puits de l'ile Eubée, en Grèce, leg. Cl. Bou). En Autriche Kovalevskiella sp. a été trouvée le long du Danube surtout dans les sédiments profonds $(1 \mathrm{~m}$ à $1,5 \mathrm{~m})$ des plages à graviers et sable de l'Ebersdorf (Melk "b"), Tulln, Wien-Floridsdorf, de "March" (Danube à l'embouchure du March) ainsi que de Wildungsmauer (dans un bras mort du Danube), dans les sédiments de la vallée du Danube à Thallern (puits F-1332), sous le lit du Piesting (0,5-0,7 $\mathrm{m}$ profondeur), à Moosbrunn II (pour la localisation des stations voir fig. I et Danielopol, 1976), enfin on l'a trouvée dans la station Kreuzgrundtraverse dans l'Eberschüttwasser, à 0,5-0,7 $\mathrm{m}$ profondeur. La découverte de Kovalevskiella sp. en Autriche élargit considérablement l'aire de répartition actuelle de ce genre (voir fig. 3). Toutefois il faut remarquer que l'espèce fossile la plus ancienne, $K$. prima provient des dépôts oligocènes d'eau dousse (Melanienton) de Marburg près de Kassel (Allemagne Fédérale), donc nettement plus au Nord-Ouest (pour plus de détails voir Carbonnel et Ritzkowski, 1969).

Les Crustacés de la famille des Parabathynellidae, à l'encontre de ceux de la famille des Bathynellidae sont peu répandus en Europe (Schminke, 1973). Il sont connus dans le bassin moyen et inférieur du Danube ainsi que dans la péninsule balkanique et en Transcaucasie, d'une part (genres Parabathynella et Hexabathynella), d'autre part dans la péninsule Ibérique, Sud de la 
France, Corse (genres Hexabathynella et Iberobathynella). Récemment des Parabathynellides ont été trouvés aussi en Autriche, dans une sonde piézométrique, à $10 \mathrm{~m}$ de distance du Piesting à Moosbrunn II et sous le lit du Schwechat, dans la même station (et profondeur) où on a récolté aussi des Bogidiella (voir ci-dessus et fig. 1).

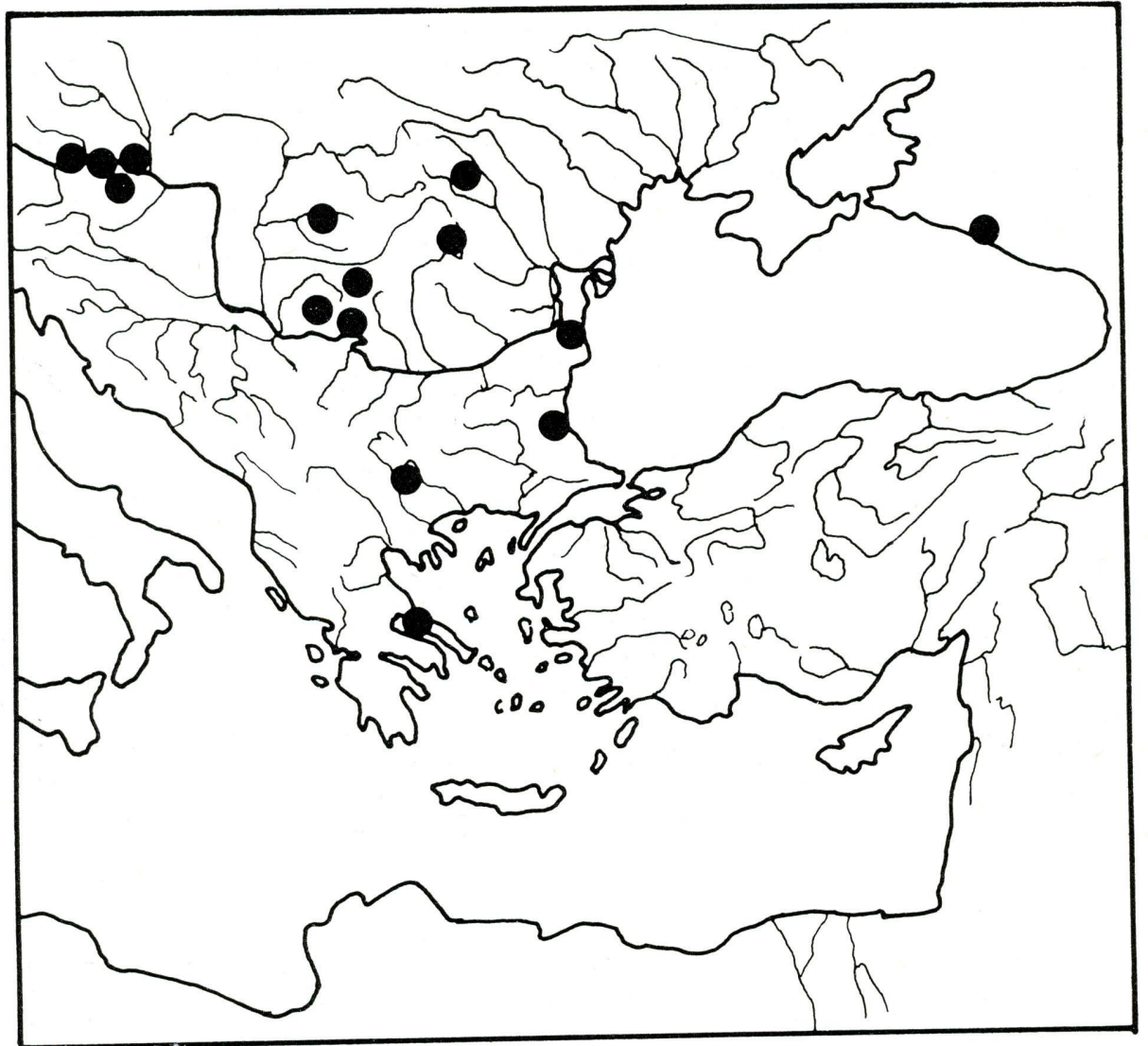

Fig. 3 - Distribution du genre Kovalevskiella ẹ Europe d'après Danielopol (1970) et données inédites.

La faune interstitielle de la Basse-Autriche, en tenant compte des connaissances actuelles (voir aussi Ruttner-Kolisko, 1955, Löffler, 1963, Kiefer, 1964 , Vornatscher, 1974), est nettement plus pauvre que la faune de la région méditerranienne. Toute une série de groupes d'animaux interstitiels connus dans la région méditerranéenne manquent. (Voir, par example, parmi les Crustacés les Isopodes des genres Stenasellus, Microparasellus, Microcerberus 
et les Amphipodes des genres Balkanella, Hadzia, Salentinella etc). Par contre la faune de Basse-Autriche contient des éléments qui n'existent pas au Nord du bassin du Danube en Europe i.e. les Microparasellides et les Parabathynellides. Les différences existants entre la faune interstitielle méditerranéenne et celle de Basse-Autriche pourraient être dues aux conditions climatiques dissemblables ayant existé durant le pléistocène dans les deux régions. Il est connu que le climat a été nettement plus rude en Europe au Nord des Alpes que dans la région méditerranéenne.

Les recherches sur la faune interstitielle de la Basse-Autriche-devront être poursuivies notamment dans certains habitats qui ont moins souffert des rigueurs climatiques pléistocènes. De tels habitats existent autour des sources thermales Bad Fischau et Bad Vöslau au Sud de Vienne.

\section{RÉSUMÉ}

La large distribution de l'Isopode interstitiel Proasellus slavus ainsi que de nouvelles stations pour les genres Troglochaetus (Archiannelida) et Bogidiella (Amphipoda). en Basse-Autriche, sont signalées. Des espèces appartenant aux genres Microcharon (Isopoda Microparasellidae), Kovalevskiella (Ostracoda, Metacyprinae) ainsi que des Parabathynellidae (Bathynellacea) sont signalées pour le première fois en Autriche.

\section{BIBLIOGRAPHIE}

CARBONNEL, G. \& RITZKOWSKI, S., 1969: Ostracodes lacustres de l'Oligocène (Melanienton) de la Hesse (Allemagne). Arch. Sc. Genève, 1: 55-82.

COINEAU, N., 1971: Les Isopodes interstitiels. Documents sur leur écologie et leur biologie. Mém. Mus. Hist. Nat. N.S., A., 64: 1-170.

CVETKOV, L., 1967: Matériaux sur les Isopodes souterrains de Bulgarie. IIMicrocharon major Karaman et Microcharon phlegetonis n.sp. C. R. Acad. Bulg. Sci., 20: 951-954.

DANIELOPOL, D., 1970: Sur la morphologie, l'origine et la répartition du genre Cordocvthere Dan. (Ostracoda, Cytheridae). Livre du Centenaire Emile G. Racovitza: $287-300$. Edit. Acad. R. S. Roumanie, Bucarest.

1976: The distribution of the fauna in the interstitial habitats of riverine sediments of the Danube and Piesting (Austria) Int. J. Speleol. 8: 23-51.

HUSSMANN, S., 1962: Ökologische und verbreitungsgeschichtliche Studien über den Archianneliden Troglochaetus heranecki Delachaux; Mitteilung über Neufunde aus den Grundwasserströmen von Donau, Ybbs, Ötz, Isar, Lahn, Ruhr, Niederrhein und Unterweser. Zool. Anz. 168: 7-10; 312-325.

LÖFFLER, H., 1967: Beiträge zur Fauna Austriaca. I. Die Ostracodenfauna Österreichs. Sitz.Ber. Öster. Akad. Wiss. Math. Nat. Kl. Abt. 1, 172: 193-211.

KIEFER, F., 1964: Zur Kenntnis der subterranen Copepoden (Crustacea) Österreichs. Ann. Naturhistor. Mus. Wien. 67: 477-485.

RUTTNER-KOLISKO, A., 1956: Rheomorpha neistwestnovae and Marinellina flagellata, zwei phylogenetisch interessante Wurmtypen aus dem Süsswasserpsammon. Österr. Z.ool. Z. 6: $55-69$.

SCHMINKE, H. K., 1973: Evolution, System und Verbreitungsgeschichte der Familie Parabathynellidae (Bathynellacea, Malacostraca). Mikrofauna Meeresboden, 24: 3-192.

SERBAN, E., 1966: Nouvelles données sur les Microparasellides (Isopodes) de Roumanie; Microcharon motasi nov. sp., Microcharon orghidani nov. sp. et Microcharon oltenicus nov. sp. Vie et Milieu, 15, 2: 341-364. 
STROUHAL, H., 1958: Asellus (Proasellus)im nördlichen Österreich (Isopoda, Asellota). Ann. Naturhistor. Mus. Wien., 62: 263-283.

VORNATSCHER, J., 1965: Amphipoda. Catalogus Faunae Austriae.8: 1-3. Springer Ver!. Wien. 1974: Die Tierwelt des Grundwassers; Leben im Dunkeln. Naturgeschichte Wiens., 2: 659-673. Jugend und Volk, Wien. 